This document is confidential and is proprietary to the American Chemical Society and its authors. Do not copy or disclose without written permission. If you have received this item in error, notify the sender and delete all copies.

\title{
Charging Ultra-nanoporous Electrodes with Size-asymmetric Ions Assisted by Apolar Solvent
}

\begin{tabular}{|r|l|}
\hline Journal: & The Journal of Physical Chemistry \\
\hline Manuscript ID & jp-2015-12730y.R1 \\
\hline Manuscript Type: & Special Issue Article \\
\hline Date Submitted by the Author: & $\mathrm{n}$ a \\
\hline Complete List of Authors: & $\begin{array}{l}\text { Rochester, Christopher; Imperial College London, Department of Physics } \\
\text { and Department of Chemistry } \\
\text { Kondrat, Svyatoslav; Forschungszentrum Julich, IBG-1: Biotechnology } \\
\text { Pruessner, Gunnar; Imperial College London, Department of Mathematics } \\
\text { Kornyshev, Alexei A. ; Imperial College London, Department of Chemistry }\end{array}$ \\
\hline
\end{tabular}




\title{
Charging Ultra-Nanoporous Electrodes with Size-Asymmetric Ions Assisted by Apolar Solvent
}

\author{
Christopher C. Rochester, ${ }^{1,2}$ Svyatoslav Kondrat, ${ }^{3}$ \\ Gunnar Pruessner, ${ }^{4}$ and Alexei A. Kornyshev ${ }^{2}$ \\ ${ }^{1}$ Department of Physics, Faculty of Natural Sciences, \\ Imperial College London, SW7 2AZ, UK \\ ${ }^{2}$ Department of Chemistry, Faculty of Natural Sciences, \\ Imperial College London, SW7 2AZ, UK \\ ${ }^{3}$ Forschungszentrum Jülich, IBG-1: Biotechnology, 52425 Jülich, Germany \\ ${ }^{4}$ Department of Mathematics, Faculty of Natural Sciences, \\ Imperial College London, SW7 2AZ, UK
}

(Dated: March 21, 2016)

\begin{abstract}
We develop a statistical theory of charging quasi single-file pores with cations and anions of different sizes as well as solvent molecules or voids. This is done by mapping the charging onto a one-dimensional Blume-Emery-Griffith model with variable coupling constants. The results are supported by three-dimensional MonteCarlo simulations in which many limitations of the theory are lifted. We explore the different ways of enhancing the energy storage which depend on the competitive adsorption of ions and solvent molecules into pores, the degree of ionophilicity and the voltage regimes accessed. We identify new solvent-related charging mechanisms and show that the solvent can play the rôle of an "ionophobic agent" effectively controlling the pore ionophobicity. In addition, we demonstrate that the ion-size asymmetry can significantly enhance the energy stored in a nanopore.
\end{abstract}

\section{INTRODUCTION}

Energy storage density can be greatly increased by the use of structured nanoporous electrodes. Such electrodes benefit both from the increase in the electrode surface area to 
volume ratio, which allows the accumulation of more charge in a given volume, and an increase in the capacitance per surface area (specific capacitance) that has been observed when ions are confined into a conductive pore that is just large enough to admit them. ${ }^{1-3}$

In extremely narrow pores the electronic charge of the pore is close enough to the incoming ions to significantly screen their electrostatic interactions. This reduces the repulsion between counterions that fill the pore upon charging and allows the accumulation of more charge at the given voltage. ${ }^{4,5}$ Such a dense packing of ions explains the increase in specific capacitance in very narrow pores ${ }^{4,6}$ and was given the name "superionic state" in one of the theoretical works ${ }^{4}$ that proposed the concept. The phenomenon has been confirmed by recent simulations. ${ }^{6-8}$

The energy stored in a capacitor is roughly proportional to the square of the voltage, so that increasing the voltage window of the capacitor will usually increase the energy density. This mainly involves an adroit choice of the electrolyte. However, if the pores are so narrow that they are saturated with counterions at relatively low voltages, further increase of voltage will have no effect on the energy storage. Such a saturation has been observed experimentally ${ }^{9}$ to occur at voltages of about $1 \mathrm{~V}$ (applied between the cathode and anode) for pores with the average pore size $\approx 0.7 \mathrm{~nm}$ and the tetraethylammonium/tetrafluoroborate ions (0.68 and $0.33 \mathrm{~nm}$ in diameter, respectively). An important practical question that we address in this work is how to increase the saturation voltage while keeping the pores sufficiently narrow to achieve high capacitances and stored energy densities.

If the electrolyte has an aqueous component, then electrolysis will occur at the electrode once a voltage of about $1.25 \mathrm{~V}$ is applied. ${ }^{10}$ This damages and ultimately destroys the capacitor. To move to higher voltages we must use a suitable non-aqueous electrolyte with a wider voltage window. This has driven interest in capacitors constructed using hydrophobic ionic liquids, some of which have a stable electrochemical window up to $6 \mathrm{~V}^{11-13}$ Steric effects between ionic molecules of vastly different sizes make this liquid state possible, moving the melting point to below room temperature. ${ }^{14,15}$ Incorporating this asymmetry in ion size is essential for bringing idealised models of capacitive charging closer to reality.

Although electrodes composed of ultra-narrow pores have obvious drawbacks in terms of power storage, such as impeding ion transport, they are a natural apex when one attempts to optimise energy density while taking advantage of the superionic effect. Motivated by this, in this paper we consider the charging of narrow cylindrical pores. The exponential 
screening of interionic electrostatic interactions inside such pores ${ }^{4,5,16,17}$ allows us to consider only nearest neighbour ion interactions and apply the corresponding statistical theory. In previous studies, our group has developed models to describe charge storage with cations and anions of the same size. ${ }^{18,19}$ Here we extend these ideas to include ion size asymmetry.

For counterions and co-ions to replace each other or solvent molecules in response to polarisation of the pore, i.e. to recharge the pore, they need to bypass each other, which in any ultranarrow, quasi-single-file pore will require some activation energy. This is possible due to thermal fluctuation of local pore radii, reorientations of anisotropic ions, and, to a lesser extent their conformational fluctuations. It is obvious from experiments that the mechanisms exists, as these pores do get charged. Of course, in the case of a true single-file pores, all ions will have to leave the pores and refill it again preferentially with counterions, but this is an extreme situation. The mechanisms of charging will affect the charging dynamics. However, the present paper is about equilibrium thermodynamics, so we do not consider these kinetic aspects. In Monte Carlo simulations we introduce a swapping mechanism, to facilitate the simulation.

\section{THEORY}

To describe charge storage in an ultra-narrow cylindrical nanopore which is so narrow to admit ions only in a single file, we will introduce a one-dimensional lattice, with $N$ sites, that runs through the centre of the pore perpendicular to its radial axis (see Figure 1). Each site on this lattice is characterised by a spin $s_{i} \in\{+1,0,-1\}$ where $i=1,2, \ldots, N$ is an integer labelling each ion in the pore. As we will further discuss below, the index $i$ labels ions in the sequence as they are laid out on the lattice. Although we refer to "sites", those do not have a fixed geometric position. The position of the ions is not a strictly linear function in $i$, but depends on the sizes of preceding ions. The lattice is therefore not a spatial, geometrical structure, but serves only the purpose of ordering the sites in a particular sequence. The spin $s_{i}=1$ represents a cation centred on the lattice site $i$, the spin $s_{i}=-1$ an anion and $s_{i}=0$ a space containing uncharged solvent or (in the present model) equivalently a void. The magnitude of the charge carried by ions of either species is assumed to be identical and equals the elementary charge $e$. A similar mapping of charge onto a lattice was used in Refs. 18 and 19 and ultimately inspired by the work of Démery et al. ${ }^{20,21}$ 


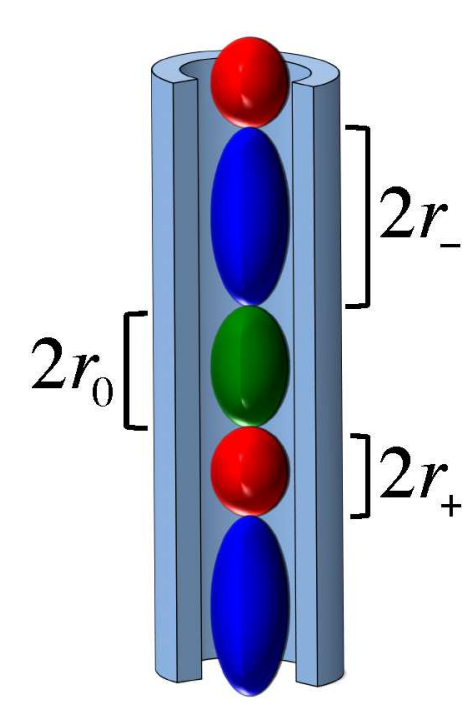

(a)

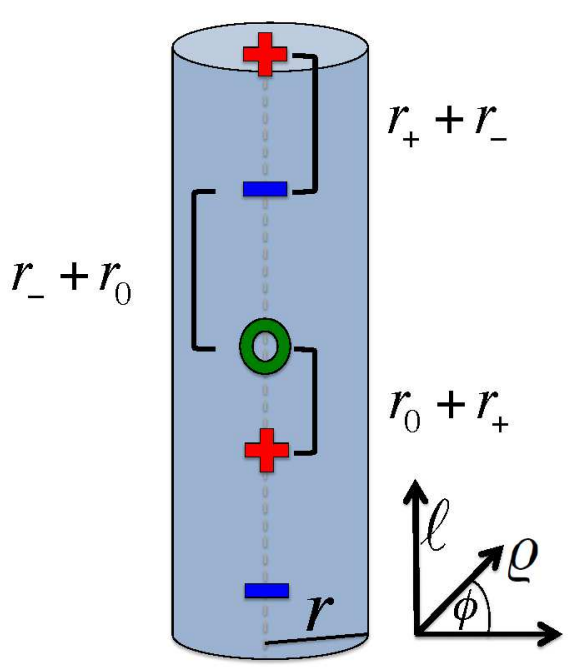

(b)

FIG. 1. A schematic representation of ions and solvent or voids of different sizes inside a cylindrical pore and the mapping of these ions to a lattice. The blue ellipsoids represent anions, red ellipsoids cations and green ellipsoids solvent or voids. The coordinate system used in the Monte-Carlo simulations, Sec. III, is shown in (b).

To incorporate the different sizes of cations, anions and solvent molecules (or voids) we allow the distance, $\ell_{i}$, separating lattice sites $s_{i}$ and $s_{i+1}$, to depend on the species occupying these neighbouring sites. Each cation that occupies $s_{i}$ or $s_{i+1}$ contributes $r_{+}$to $\ell_{i}$, while anions contribute $r_{-}$and solvent molecules or voids $r_{0}$. For example, two adjacent lattice sites occupied by an anion and a void will be separated by $r_{-}+r_{0}$. We interpret $r_{+}$and $r_{-}$ as the radii of a cation and an anion, respectively, while $r_{0}$ is the radius of a solvent molecule or void (see Fig. 1).

Electrostatic interactions between ions inside the pore are screened by the metallic electrons of the pore walls. We assume this screening to be ideal, so that interactions beyond nearest neighbours can be neglected. The electrostatic interaction energy of two monovalent point charges separated by a distance $\ell$ in the centre of an ideally screening metallic cylinder of radius $r$ is approximately given by ${ }^{5,18,19,22}$ (in units of $k_{B} T$ which we will use throughout)

$$
J(\ell, r) \simeq \frac{3.08 L_{B}}{r} \exp (-2.4 \ell / r), \quad \ell \gg r,
$$

where $L_{B}=e^{2} /\left(\epsilon k_{\mathrm{B}} T\right)$ is the Bjerrum length. Here $e$ is the elementary charge and $\epsilon$ the 
dielectric constant in the pore. We emphasise that Eq. (1) is an approximate expression for the ion-ion interaction potential (for small pore radii $r \ll \ell$ ) that results from the exact analytical solution of the Poisson equation for a single point charge confined in a metallic cylindrical pore (cf. Eq. (10)).

The (single) charge of an ion in the pore is assumed to be located at the centre of the ion. The interaction energy between nearest neighbouring charges, $s_{i}$ and $s_{i+1}$, is given by $J\left(\ell_{i}, r\right)$. The interaction $J_{s s^{\prime}}$ between two ions of species $s$ and $s^{\prime}$ is a function of the distance and the pore radius, $J_{s s^{\prime}}=J\left(r_{s}+r_{s^{\prime}}, r\right)$, for example $J_{+-}=J\left(r_{+}+r_{-}, r\right)$. A solvent molecule or a void has no electrostatic interaction with its neighbours and any ion dipole interactions are neglected. As electrostatic interactions inside the pore decay exponentially in the charge separation, with a decay length smaller than the shortest distance between any two ions, we will neglect all but nearest neighbour interactions.

The pore itself is polarised by a potential, $h$. In the following, we parameterise the free energy of transfer of ions from the bulk into the pore by $w_{+}$and $w_{-}$for cations and anions respectively. There are various contributions to these quantities: Energetic ones, such as electrostatic solvation energy in the bulk, interaction with images in the pore, van-der-Waals interactions, and entropic ones, related to the difference in the ion confinement in solvation shell in the bulk versus their localisation in the pore. These values are exceedingly difficult to calculate form first principles, not least as they are highly system-specific. Thus, $w_{ \pm}$ are to be considered as phenomenological parameters. The Hamiltonian, $H$, and partition function, $Z$, are therefore

$$
H=\sum_{\langle i, j\rangle} J_{s_{i}, s_{j}} s_{i} s_{j}+\sum_{i} h_{s_{i}} s_{i}, \quad Z=\sum_{\{s\}} \mathrm{e}^{-H}
$$

where $\langle i, j\rangle$ represents the sum over nearest neighbours, $\{s\}$ the sum over all ion configurations and $h_{s_{ \pm}}=e h \mp w_{ \pm}$is the net energy of transfer of a single cation or anion taken out of the bulk and placed into the pore. This Hamiltonian is similar to a modified BlumeEmery-Griffiths model in the theory of magnetism ${ }^{23}$ where $J_{s_{i}, s_{j}}$ would play the role of the spin-spin coupling and $h_{s_{i}}$ a spin-dependent magnetic field coupling.

To obtain an analytical expression for the partition sum, Eq. (2), we consider the transfer 
matrix

$$
\underline{\mathrm{T}}=\left(\begin{array}{ccc}
\exp \left(-J_{++}-h_{+}\right) & \exp \left(-\frac{1}{2} h_{+}\right) & \exp \left(J_{+-}-\frac{1}{2}\left(h_{+}-h_{-}\right)\right) \\
\exp \left(-\frac{1}{2} h_{+}\right) & 1 & \exp \left(\frac{1}{2} h_{-}\right) \\
\exp \left(J_{+-}-\frac{1}{2}\left(h_{+}-h_{-}\right)\right) & \exp \left(\frac{1}{2} h_{-}\right) & \exp \left(-J_{--}+h_{-}\right)
\end{array}\right)
$$

which is symmetric and thus has real eigenvalues $\lambda_{+}, \lambda_{0}, \lambda_{-}$, so that $Z=\lambda_{+}^{N}+\lambda_{0}^{N}+\lambda_{-}^{N}$ in a system with periodic boundary conditions.

Irrespective of the boundary conditions, in the thermodynamic limit the Helmholtz free energy density (in units of $k_{B} T$ ), $f$, is determined by the largest eigenvalue $\lambda_{+}$of the transfer matrix,

$$
f=-\lim _{N \rightarrow \infty} \frac{1}{N} \ln Z=-\ln \lambda_{+} .
$$

The closed form of this eigenvalue is ungainly, as expected for eigenvalues of matrices larger than $2 \times 2$ and is therefore not reproduced in the present work. It can be found via methods detailed in Refs. 24 and 25.

To obtain thermodynamic observables we differentiate the free energy density in (4) with respect to the relevant conjugate variable. The density of cations, $\left\langle\rho_{+}\right\rangle$, anions, $\left\langle\rho_{-}\right\rangle$, and solvent (or voids) $\left\langle\rho_{0}\right\rangle$ in the pore, are obtained as

$$
\left\langle\rho_{+}\right\rangle=\frac{\partial f}{\partial w_{+}}, \quad\left\langle\rho_{-}\right\rangle=\frac{\partial f}{\partial w_{-}}, \quad\left\langle\rho_{0}\right\rangle=1-\left\langle\rho_{+}\right\rangle-\left\langle\rho_{-}\right\rangle .
$$

The charge per unit area of the pore wall is therefore

$$
\sigma=\frac{\left\langle\rho_{+}\right\rangle+\left\langle\rho_{-}\right\rangle}{2 \pi\langle\ell\rangle} e
$$

where

$$
\langle\ell\rangle=2\left(r_{+}\left\langle\rho_{+}\right\rangle+r_{-}\left\langle\rho_{-}\right\rangle+r_{0}\left\langle\rho_{0}\right\rangle\right)
$$

is the average lattice spacing. The differential capacitance of a pore is given by

$$
C=\frac{\partial \sigma}{\partial h}
$$

so that the total energy stored in the pore between zero potential and $h$ is given by the integral

$$
E(h)=\int_{0}^{h} \mu C(\mu) \mathrm{d} \mu
$$

which can be calculated numerically once the capacitance is found. 


\section{MONTE-CARLO SIMULATIONS}

Complementary Monte-Carlo simulations were performed to validate the various approximations in the above theory. Importantly, in the simulations the molecules within the pore are not constrained to sit at the centre of the pore or be densely packed. These simulations are similar to those performed in Ref. 7, which consider the charging of slit pores, and Ref. 19, which studies the charging of cylindrical pores. In the following, we will only summarise the simulation method and refer the reader to the aforementioned publications for further details.

Hard sphere ions of different sizes are placed inside an ideally screening cylindrical pore. These ions have a chemical potential, interact with each other as well as with their own image charges. The ions cannot overlap and two monovalent ions centred at $(0, \bar{\varrho}, 0)$ and $(\ell, \varrho, \phi)$ have an interaction energy of

$$
J(\ell, \varrho, \bar{\varrho}, \phi)=\frac{e^{2}}{\epsilon} \frac{2}{r} \sum_{m=0}^{\infty} a_{m} \cos m \phi \sum_{n=1}^{\infty} \frac{J_{m}\left(k_{n m} \bar{\varrho} / r\right) J_{m}\left(k_{n m} \varrho / r\right)}{k_{n m} Y_{m+1}\left(k_{n m}\right)^{2}} e^{-k_{n m}|\ell / r|}
$$

where $a_{m}=1$ if $m=0$ and $a_{m}=2$ otherwise, $J_{m}(x)$ and $Y_{m}(x)$ are the Bessel functions of the first and second kind and $k_{n m}$ is the $\mathrm{n}^{\text {th }}$ positive root of $J_{m}(x)$. In the special case of $\bar{\varrho}=\varrho=0$ this formula reduces to the approximation Eq. (1). ${ }^{26}$ The interaction energy between a charge located at $(0, \bar{\varrho}, 0)$ and its own induced image charges is given by

$$
\begin{aligned}
U(\bar{\varrho}, r)=-\frac{e^{2}}{2 \pi^{2} r \epsilon} \sum_{m=0}^{\infty} a_{m} \int_{0}^{2 \pi} \mathrm{d} \phi & \cos m \phi \\
& \times \int_{0}^{\infty} \mathrm{d} \xi \frac{I_{m}(\xi \bar{\varrho} / r)}{I_{m}(\xi)} K_{0}\left(\frac{\xi}{r} \sqrt{\bar{\varrho}^{2}+r^{2}-2 r \bar{\varrho} \cos \phi}\right)
\end{aligned}
$$

where $I_{m}(x)$ and $K_{m}(x)$ are the modified (or hyperbolic) Bessel functions of the first and second kind respectively. Molecules are forced into the pore by a transfer energy, $w_{ \pm}^{\text {sim }}$, for cations and anions and $w_{0}^{\text {sim }}$ for a solvent (as in the lattice model the signs are chosen such that the larger $w_{0}^{\text {sim }}$ or $w_{ \pm}^{\text {sim }}$ the more solvent- or ionophilic is the pore, respectively). The transfer energy $w_{ \pm}$introduced in the theory above is related to these transfer energies via

$$
w_{ \pm}=w_{ \pm}^{\mathrm{sim}}-U(\bar{\varrho}=0, r)-w_{0}^{\mathrm{sim}}
$$

assuming that ions position themselves on the symmetry axis of the pore; for instance, for a pore of radius $r=0.375 \mathrm{~nm}$ we have $U \approx-26.8 k_{B} T$ at room temperature. In simulations 
we distinguish between a solvent molecule and a void and therefore we have to introduce the transfer energy of a solvent, $w_{0}^{\text {sim }}$, which had to be fitted to compare with the theory. We choose $w_{0}^{\text {sim }}$ such that a pore is nearly fully occupied at any voltage either by ions or by solvent in correspondence to the theoretical model above, which also correspond to the experimental conditions in most cases (see e.g. Refs. 1, 2, 27-29). However, Eq. (12) does not give a satisfactory quantitative agreement between theory and simulations, most likely due to the lateral entropy of all molecules and the shift in the interaction potentials (ions in simulations are free to reside near the pore wall and are not restricted to the middle axis as in the theory). Both effects are ignored in the theory but would modify Eq. (12). Anticipating the discussion below, the qualitative agreement between the theory and simulations is excellent, nevertheless.

Interaction potentials (10) and (11) have been implemented in MCCCS Towhee simulation package. ${ }^{30,31}$ Our system constitutes a single cylindrical pore of a specified width which was $25 \mathrm{~nm}$ long and had periodic boundary conditions applied along the longitudinal direction of the pore. The grand-canonical Monte-Carlo simulations consisted of the standard Widom insertion/deletion ${ }^{32}$ and translational moves. In addition, we allowed for a spontaneous swap of molecule species, whereby a randomly picked molecule is replaced by a molecule of different species provided it fits into the emptied space. ${ }^{7}$ The total number of simulation steps was $5 \cdot 10^{6}$ to $10^{7}$ in the equilibration runs and $2 \cdot 10^{7}$ in the production runs. To obtain better results in some cases we performed a second round of simulations starting from the saved molecular configurations of previous runs.

\section{RESULTS}

Our theory allows for the energetic costs of ions entering the pore $\left(w_{ \pm}\right)$to be speciesdependent. However, the symmetric case $w_{+}=w_{-}$is more convenient to work with. In the following, we will express all results in terms of a single transfer energy $\bar{w}$, rather than $w_{-}$ and $w_{+}$individually. This does not imply a loss of generality, as it turns out, because the case $w_{+} \neq w_{-}$is still covered by choosing

$$
\bar{w}=\frac{1}{2}\left(w_{+}+w_{-}\right)
$$


and changing the potential applied to the pore walls according to

$$
h \longrightarrow h-\frac{w_{+}-w_{-}}{2 e} .
$$

The effect of asymmetric transfer energy therefore just causes a shift of the point of zero charge, so that the pore favours one ion species over another. Henceforth, we will express our results in terms of $\bar{w}$ and $h$, bearing in mind that in the case of $w_{+} \neq w_{-}$, the mean transfer energy is determined by (13) and the potential $h$ has to be shifted according to (14)

Although asymmetric transfer energies result only in a linear shift in the potential, Eq. (14), and the differential capacitance as a function of potential, it has a profoundly greater affect on the energy stored in the pore, Eq. (9). Any difference in the transfer energies that favours coions will shift the potential linearly, Eq. (14), producing a non-linear change in the energy stored, viz. any bias in favour of coions will create an obstacle to charging and will increase the total energy stored in the pore at large voltages. ${ }^{33}$ Correspondingly, any bias in favour of counterions will decrease the total stored energy for large voltages.

We note finally that it is not trivial to relate the energies of transfer $w_{ \pm}$or $\bar{w}$ of the theory or $w_{ \pm}^{\text {sim }}$ of simulations (and hence the chemical potentials) to quantities measured directly in experiments. However, a combined molecular-dynamics and quantum-density-functional study suggests that $w_{ \pm}^{\text {sim }}$ can vary from about $-230 k_{B} T$ to $61 k_{B} T$ depending on the pore radius and an ionic liquid used (see Table 5 of Ref. 34 and note that our transfer energy differs by sign from the energies calculated there). The values of $w_{ \pm}^{\text {sim }}$ and $\bar{w}$ taken in the present work fall well within this range.

\section{A. Effect of the solvent}

The competitive absorption of solvent and ions into the pore at a given voltage is dependent on the transfer energies of the various molecules in the system. If the transfer energies are such that solvent molecules are present in the pore of a nonpolarised electrode, as in the case of figures 2 and $3,^{35}$ then the solvent acts as a blocker, an obstacle that must be removed from the pore before charging with ions can take place. This will make the pore effectively more ionophobic and enlarge the voltage range within which there is no saturation of counterions in the pore. Interestingly, smaller solvent is a better choice to increase ionophobicity (Fig. 2). This is because for two solvents with the same transfer energy, a 
larger entropic barrier must be overcome by a larger solvent to enter the pore, which also means that the larger solvent can be more easily removed from the pore.

If no solvent is present in the pore at zero voltage, it is possible, but not necessary, for it to enter the pore at certain voltages to act as a buffer between counterions, thus aiding charging, as discussed below ( $c f$. Fig. 5).

In a recent NMR study ${ }^{29}$ Forse et al have compared charging of $\mathrm{Pyr}_{13}$ TFSI ionic liquids in YP50F activated carbon electrodes with and without acetonitrile as a solvent. They observed that there is about $30 \%$ less ionic liquid inside the carbon pores in the presence of solvent $(1.8 \mathrm{M})$, even though the total pore occupancy increased by $20 \%$. Remarkably, their results suggest that the (in-pore) ion diffusion is faster in a solvent-rich system, which is consistent with recent molecular dynamics simulations ${ }^{36}$ suggesting an enhanced ion diffusion in more ionophobic pores. Thus, although the solvent in the present work is apolar, unlike acetonitrile, our results are in line with the experimental findings in that the solvent can effectively increase pore ionophobicity. 


\section{B. Charging pores with symmetric ions}

Figures 3 and 4 show nanopore charging with ions of equal size. As expected the plots are symmetric about zero voltage, $h=0$. Capacitance can have four (Fig. 3c) or two (Fig. 4a) peaks depending on the ionophobicity. These peaks reflect various charging mechanisms which we discuss in Section IV D.

Figure 3 allows a comparison between the molecular densities obtained in the theory and in Monte-Carlo simulations. Although we had to adjust the values of the transfer energies, as discussed in Sec. III, the densities found are in excellent qualitative agreement.

As mentioned in Sec. IV A, the presence of solvent at low voltages makes pores effectively more ionophobobic, but otherwise a system with apolar solvent and symmetric ions behaves qualitatively similar to the otherwise same system without solvent (highly polar solvents can have a more dramatic effect on the capacitance, however, see Ref. [37]). Since the latter setup has been extensively studied by our group ${ }^{18,19}$ and by others, ${ }^{6,38-40}$ we shall not discuss it in detail here. We recall only that the ionophobicity of pores can lead to much higher energies stored at high voltages compared to conventional ionophilic pores. ${ }^{33}$ For instance, at an applied potential of $20 k_{B} T / e$ (about $0.5 \mathrm{~V}$ at room temperature) the energy stored in an ionophobic pore $\left(\bar{w}=-10 k_{B} T\right)$ is almost tripled as compared to the ionophilic pore with $\bar{w}=0$ (see Fig. 4b).

\section{Size-asymmetric ionic liquids}

The differential capacitance, $C(h)$, changes drastically as the size of the anion decreases (Figures 5 and 6). The peaks in $C(h)$ are not any more symmetric with respect to $h=$ 0 , and the capacitances reach much higher values at these peaks (compare Fig. 3c with Fig. 5c). These changes in capacitance are reflected in the energy stored in a pore which is much greater when the pore is charged with smaller counterions (see Fig. 6b, which has a different energy scale compared to Fig. 4b). Such high stored energies are achieved only for positive voltages, however, and hence using a symmetric two-electrode electrochemical cell and $V \rightarrow-V \rightarrow V \cdots$ cycling would diminish this effect. Thus to fully benefit from size-asymmetry a clever design of asymmetric electrodes and a cycling $V \rightarrow 0 \rightarrow V \cdots$ are necessary. Interestingly, the latter can also be beneficial from a point of view of kinetics. ${ }^{41}$ 


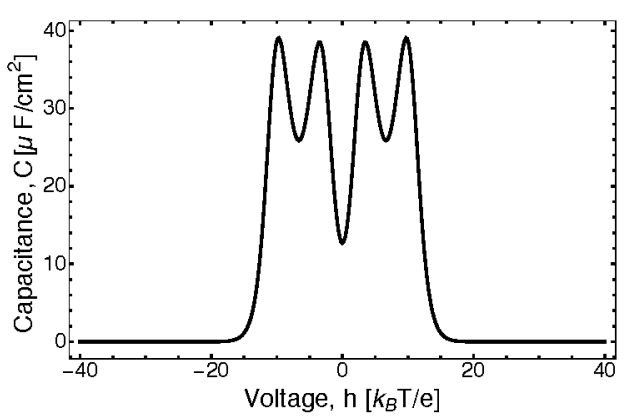

(c) Theory (differential capacitance)

FIG. 3. Comparison between Monte-Carlo (a) and theory (b) of the molecular densities for a pore charged with molecules of the same size. Both theory and simulations demonstrate expulsion of solvent molecules and their replacement with counterions as the electrode polarisation increases ( $k_{\mathrm{B}} T / e \approx 25.6 \mathrm{mV}$ at room temperature). (c) Differential capacitance curve predicted by the theory. [Parameters: Ion and solvent radii $r_{+}=r_{-}=r_{0}=0.35 \mathrm{~nm}$, pore radius $r=0.375 \mathrm{~nm}$, transfer energy $\bar{w}=-4 k_{\mathrm{B}} T$. The same parameters are applied in Monte-Carlo simulations, which uses transfer energies $w_{ \pm}^{\mathrm{sim}}=-18 k_{B} T$ and $\mu_{0}^{\mathrm{sim}}=15 k_{B} T$ at temperature $333 \mathrm{~K}$ and dielectric constant in the pore $\varepsilon=2.42$. Note that $\bar{w}$ and $w_{ \pm}^{\text {sim }}$ and $w_{0}^{\text {sim }}$ are related by Eq. (12).] 


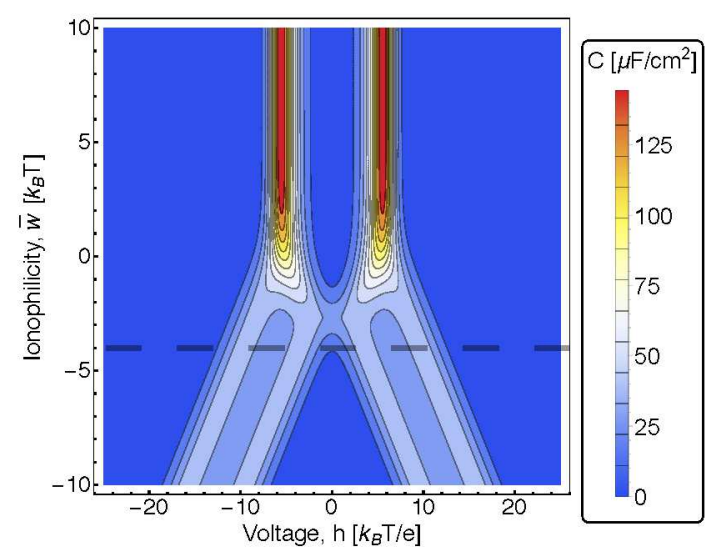

(a) Differential Capacitance

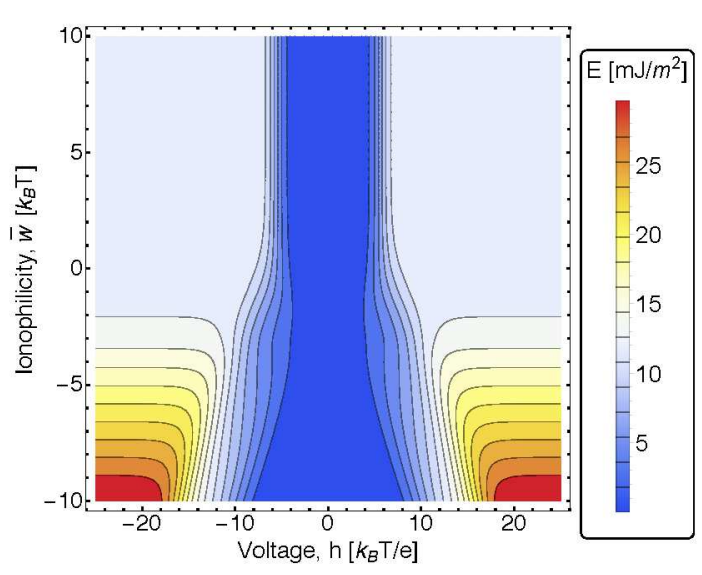

(b) Energy Stored

FIG. 4. Contour plots of (a) the differential capacitance and (b) the corresponding stored energy of a pore charged with cations and anions of the same size. The applied voltage is measured in units of $k_{\mathrm{B}} T / e \approx 25.6 \mathrm{mV}$ at room temperature. The horizontal grey broken line in the differential capacitance contour map (a) marks the cross section of constant $\bar{w}$ corresponding to the capacitance-voltage profile in figure Fig. 3c. Strongly ionophilic pores correspond to large positive $\bar{w}$. Parameters as in Figure 3.

Increasing the ionophobicity of the pore increases the maximum energy that can be stored before saturation of the pore with counterions. While ionophobic pores have the potential to increase the total energy stored in the pore at high voltages, at low voltages a lower ionophobicity or even ionophilicity might be required to maximise the energy stored (Figures $4 \mathrm{~b}$ and $6 \mathrm{~b}$ ).

\section{Charging Mechanisms}

Three independent mechanisms have been identified for charging nanopores with neat ionic liquids: ${ }^{27,28,33}$ (i) Swapping of coions for counterions, (ii) electrosorption of counterions, and (iii) desorption of coions. Adding solvent leads to more complex and richer charging behaviour with two additional charging mechanisms: (iv) solvent swapping with coions, which themselves are forced out of the pore at higher voltages and replaced with counterions, and $(\mathrm{v})$ direct swapping of solvent with counterions without the inclusion of coions in the pore at any voltage. The transfer energy $(\bar{w})$ determines which charging mechanisms takes place. 


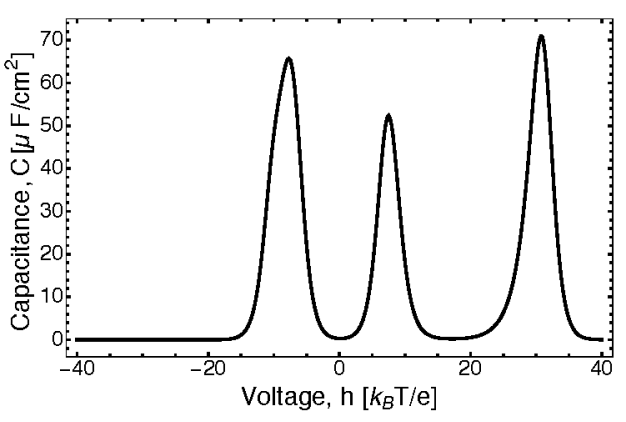

(c) Theory (differential capacitance)

FIG. 5. Comparison between Monte-Carlo (a) and theory (b) of the molecular densities for a pore charged with ions of differing size. Both theory and simulations demonstrate expulsion of solvent molecules and their replacement with counterions as the electrode polarisation increases $\left(k_{\mathrm{B}} T / e \approx 25.6 \mathrm{mV}\right.$ at room temperature). (c) Differential capacitance curve predicted by the theory. [Parameters: Cation radius $r_{+}=0.35 \mathrm{~nm}$, anion $r_{-}=0.225 \mathrm{~mm}$, solvent $r_{0}=0.35 \mathrm{~nm}$ and pore $r=0.375 \mathrm{~nm}$, transfer energy $\bar{w}=-4 k_{\mathrm{B}} T$. The same parameters are applied in Monte-Carlo simulations, which uses transfer energies $w_{ \pm}^{\text {sim }}=-18 k_{B} T$ and $w_{0}^{\text {sim }}=15 k_{B} T$ at temperature $333 \mathrm{~K}$ and dielectric constant in the pore $\varepsilon=2.42$. Note that $\bar{w}$ and $w_{ \pm}^{\text {sim }}$ and $w_{0}^{\text {sim }}$ are related by Eq. (12).] 


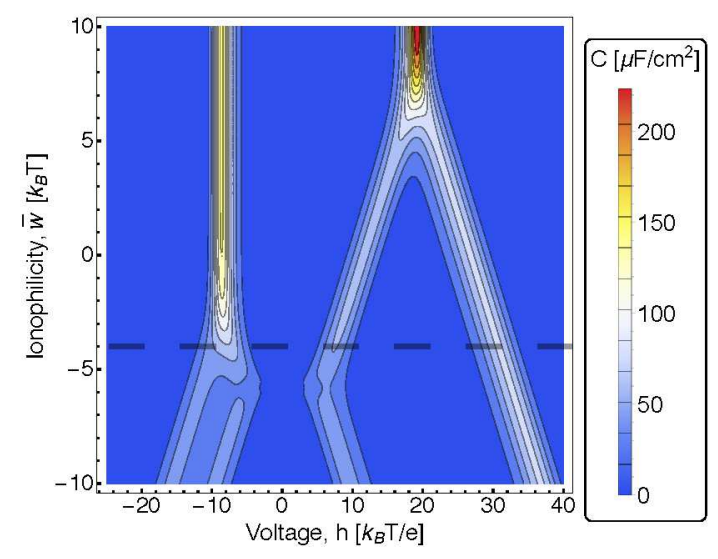

(a) Differential Capacitance

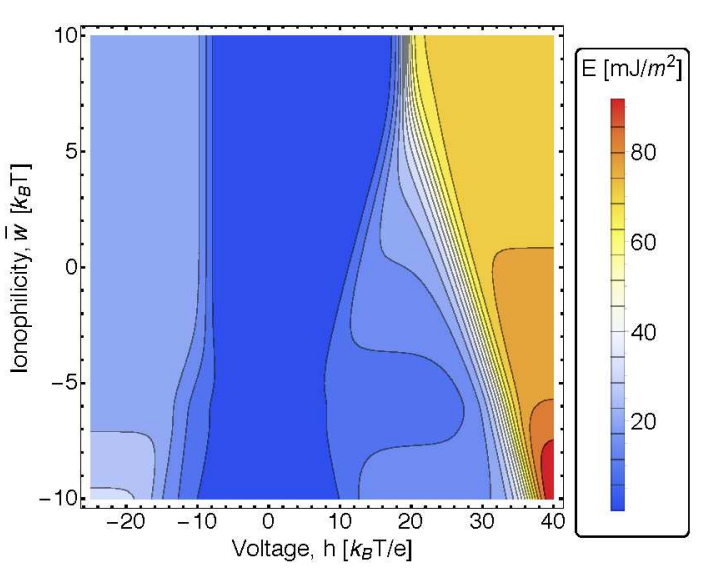

(b) Energy Stored

FIG. 6. Contour plots of (a) the differential capacitance and (b) the corresponding stored energy of a pore charged with cations and anions of different size. The applied voltage is measured in units of $k_{\mathrm{B}} T / e \approx 25.6 \mathrm{mV}$ at room temperature. The horizontal gray broken line in the differential capacitance contour map (a) marks the cross section of constant $\bar{w}$ corresponding to the capacitance-voltage profile shown in figure Fig. 5c. Strongly ionophobic pores correspond to large negative $\bar{w}$. Parameters as in Fig. 5 .

These charging mechanisms corresponding to different ionophobicities of the pore at different voltages are listed in Table I. The various thresholds in this table have been calculated from the peaks in the capacitance, and the charging mechanisms identified by looking at how the equilibrium ion and solvent densities change with the applied potential. Note that we do not observe isolated electrosorption and desorption processes as they are always assisted by the solvent. This is because the pores considered here are almost completely occupied by ions or solvent even with no voltage applied.

\section{DISCUSSION}

We have developed a lattice model of charge storage in cylindrical nanopores charged with cations and anions of different sizes in the presence of solvent. Our theory also covers the situation of an empty pore with gaps between ions. We have validated the results by grand-canonical Monte-Carlo simulations of the same system. In addition to the three known charging mechanisms ${ }^{27,28,33}$ we have identified two further solvent-related charging 


\begin{tabular}{|c|c|c|c|c|}
\hline Ionophobicity & Transfer energy & Thresholds $\left(e h^{*}\right)$ & Mechanisms & Type \\
\hline \multirow{2}{*}{$\begin{array}{l}\text { Strongly } \\
\text { Ionophilic }\end{array}$} & $\bar{w} \gg J_{--}-J_{+-}$ & $\left(J_{+-}+J_{--}\right)$ & $-+-\longrightarrow---$ & \multirow{2}{*}{$\begin{array}{c}\text { (i) coion-counterion } \\
\text { swapping }\end{array}$} \\
\hline & $\bar{w} \gg J_{++}-J_{+-}$ & $-\left(J_{+-}+J_{++}\right)$ & $+-+\longrightarrow+++$ & \\
\hline \multirow{2}{*}{$\begin{array}{c}\text { Weakly } \\
\text { Ionophobic }\end{array}$} & $-J_{+-} \leq \bar{w}<J_{--}-J_{+-}$ & $\begin{array}{l}\left(2 J_{+-}+\bar{w}\right) \\
\left(2 J_{--}-\bar{w}\right)\end{array}$ & $\left|\begin{array}{l}-+-\longrightarrow-\circ- \\
-\circ-\longrightarrow---\end{array}\right|$ & \multirow{2}{*}{$\begin{array}{l}\text { (iv) coion-solvent- } \\
\text {-counterion swapping }\end{array}$} \\
\hline & $-J_{+-} \leq \bar{w}<J_{++}-J_{+-}$ & $\begin{array}{l}-\left(2 J_{+-}+\bar{w}\right) \\
-\left(2 J_{++}-\bar{w}\right)\end{array}$ & $\begin{array}{l}+-+\longrightarrow+\circ+ \\
+\circ+\longrightarrow+++\end{array} \mid$ & \\
\hline \multirow{2}{*}{$\begin{array}{c}\text { Strongly } \\
\text { Ionophobic }\end{array}$} & \multirow{2}{*}{$\bar{w}<-J_{+-}$} & $\begin{array}{c}-\bar{w} \\
\left(2 J_{--}-\bar{w}\right)\end{array}$ & $\begin{array}{l}\circ \circ \circ \longrightarrow \circ-\circ \\
\circ-\circ \longrightarrow---\end{array}$ & \multirow{2}{*}{$\begin{array}{c}\text { (v) solvent-counterion } \\
\text { swapping }\end{array}$} \\
\hline & & $\begin{array}{c}\bar{w} \\
-\left(2 J_{++}-\bar{w}\right)\end{array}$ & $\begin{array}{c}\circ \circ \circ \longrightarrow \circ+\circ \\
\circ+\circ \longrightarrow+++\end{array}$ & \\
\hline
\end{tabular}

TABLE I. Summary of the charging mechanisms in pores of various ionophobicities charged with ions of different sizes and solvent. The threshold refers to the voltage needed to activate the charging process. Cations, anions and solvent are graphically represented by,+- and $\circ$ respectively. Various types of charging mechanisms are described in the text. We do not observe isolated electrosorption of counterions (type (ii)) and desorption of coions (type (iii)), most likely because we consider pores that are fully occupied by ions and/or solvent even at zero voltage.

mechanisms in the present setup (see Section IV D, in particular Tab. I). The ionophobicity of a pore decides the prevailing charging mechanism.

The notion of ionophobicity of pores has been introduced and discussed in our previous works, where we have shown that ionophobic pores can store more energy ${ }^{19,33}$ and provide much higher power densities ${ }^{36,42}$ than conventional ionophilic pores. In the present work, we have demonstrated that this property of pores can be effectively controlled by using solvents with different wetting abilities (Fig. 2). Although the effect of so-controlled ionophilicity on the stored energy is apparent, it remains to be seen how the presence of a solvent influences the charging dynamics and hence power density.

The total energy that can be stored in corresponding ionophilic and weakly ionophobic pores is comparable, but strongly ionophobic pores can store more energy than ionophilic and weakly ionophobic pores if charged at high voltages (Fig. 4). Interestingly, however, less ionophobic pores store more energy at lower voltages. To optimise the total energy stored in 
one polarity of the pore one needs to use a smaller counterion, make the pore favour coions or increase the ionophobicity so that counterions will have to be forced into the pore (Fig. 6). Some of these measures may have a detrimental effect to energy storage on the oppositely charged electrode. Obviously, when engineering a supercapacitor the gains at one electrode have to be offset against the losses at the other electrode.

Solvent molecules in our model played a role of a hard core buffer. They also contribute to the effective dielectric constant of the interior of the pore. The latter is determined by the electronic polarisability of the ions and electronic and vibrational polarisability of solvent molecules; in principle, even in a quasi-one-dimensional pore, some librational contribution may also be possible. Whereas the electronic-polarisability-related dielectric constant is typically of the order of 2 , the vibrational and librational constant can easily reach 4 to 5 . In that case the effective dielectric constant would depend on the composition of pore filling (the more solvent inside, the higher the dielectric constant). In all calculations above we have considered only electronic degrees of freedom, and thus the effect of the composition of the liquid inside the pore on the effective dielectric constant will be minor. A more detailed description of the 'high frequency' polarisability of the ions may, generally, be important, and will be considered in future work.

Charging an electrode is always accompanied by the effect of electrowetting, as highpolarity material tends to wet the surface of the electrode at the expense of the less polar one. It would thus be expected that by polarising the electrode the counterions will tend to fill the pore and non-polar molecules will leave it. This effect is of course covered by our theory. Through parameters $w_{ \pm}$we capture not the electrowetting effect but pure wetting. The wetting capacity of the surface with respect to the solvent and the ions is highly dependent on the electrode material and the nature of the ions. In a first approximation it can be tested through the measurement of the wetting angle on a flat surface of a non-polarised electrode, for a droplet of a solvent or of an ionic liquid. The wetting properties inside the nanopore may be different from those of a macropscopic flat surface. So the question, "who" is inside a non-polarised pore remains subject of debate and ongoing experiments, such as NMR experiments of the Clare Grey group ${ }^{27-29,43}$, the electrochemical quartz crystal microbalance experiments by the Levi ${ }^{44-46}$ and Simon ${ }^{28,47,48}$ groups, and the wetting experiments by the Gogotsi group ${ }^{49}$.

In the present work we deliberately explored also the extreme cases for the wetting 
ability of the pores with respect to ions and solvent molecules, in order to reveal interesting, non-trivial scenarios which might be possible to realise in future electrode materials and electrolyte combinations.

Finally, we have considered tight cylindrical pores that have evident drawbacks from the point of view of power density. Thus, they do not seem to be very useful and either wider cylindrical pores or slit pores shall be used in practical applications. However, these tight cylindrical pores have given us an excellent opportunity to analyse charging processes analytically, while they also show features akin of more practical slit nanopores. There we expect qualitatively similar ionophobicity- and voltage-dependent behaviour of the capacitance and stored energy in the absence of solvent. ${ }^{4,7,18,19,33}$ It will be interesting to look at the effect of a solvent in slit pores, whose analysis will most likely involve approximate mean-field type theories or simulations.

\section{ACKNOWLEDGEMENTS}

CR acknowledges the support of the Center for Doctoral Training on Theory and Simulation of Materials at Imperial College London, which is funded by the EPSRC under grant number EP/G036888/1. We are grateful to Alexander Forse for fruitful suggestions and comments.

\section{BIBLIOGRAPHY}

1 J. Chmiola, G. Yushin, Y. Gogotsi, C. Portet, P. Simon, and P. L. Taberna, "Anomalous increase in carbon capacitance at pore sizes less than 1 nanometer," Science, vol. 313, no. 5794, pp. 1760-1763, 2006.

${ }^{2}$ E. Raymundo-Piñero, K. Kierczek, J. Machnikowski, and F. Béguin, "Relationship between the nanoporous texture of activated carbons and their capacitance properties in different electrolytes," Carbon, vol. 44, pp. 2498-2507, 2006.

3 C. Largeot, C. Portet, J. Chmiola, P. Taberna, Y. Gogotsi, and P. Simon, "Relation between the ion size and pore size for an electric double-layer capacitor," J. Am. Chem. Soc., vol. 130, 
no. 9, pp. 2730-2731, 2008.

${ }^{4}$ S. Kondrat and A. Kornyshev, "Superionic state in double-layer capacitors with nanoporous electrodes," J. Phys.-Condens. Matter, vol. 23, no. 2, p. 022201, 2011.

${ }^{5}$ C. C. Rochester, A. A. Lee, G. Pruessner, and A. A. Kornyshev, "Interionic interactions in conducting nanoconfinement," ChemPhysChem, vol. 14, no. 18, pp. 4121-4125, 2013.

${ }^{6}$ B. Skinner, T. Chen, M. Loth, and B. I. Shklovskii, "Theory of volumetric capacitance of an electric double-layer supercapacitor," Phys. Rev. E, vol. 83, no. 5, p. 056102, 2011.

7 S. Kondrat, N. Georgi, M. V. Fedorov, and A. A. Kornyshev, "A superionic state in nano-porous double-layer capacitors: Insights from Monte Carlo simulations," Phys. Chem. Chem. Phys., vol. 13, no. 23, pp. 11359-11366, 2011.

8 C. Merlet, B. Rotenberg, P. A. Madden, P. L. Taberna, P. Simon, Y. Gogotsi, and M. Salanne, "On the molecular origin of supercapacitance in nanoporous carbon electrodes," Nat. Mater., vol. 11, no. 4, pp. 306-310, 2012.

9 R. Mysyk, E. Raymundo-Piñero, and F. Béguin, "Saturation of subnanometer pores in an electric double-layer capacitor," Electrochem. Comm., vol. 11, pp. 554-556, 2009.

10 P. W. Atkins and J. De Paula, Atkins' Physical Chemistry. Oxford University Press: Oxford, UK, 2006.

11 P. Bonhote, A. Dias, N. Papageorgiou, K. Kalyanasundaram, and M. Grätzel, "Hydrophobic, highly conductive ambient-temperature molten salts," Inorg. Chem., vol. 35, no. 5, pp. 1168 $1178,1996$.

12 M. Hayyan, F. S. Mjalli, M. A. Hashim, I. M. AlNashef, and T. X. Mei, "Investigating the electrochemical windows of ionic liquids," J. Ind. Eng. Chem., vol. 19, no. 1, pp. 106-112, 2013.

13 M. Armand, F. Endres, D. R. MacFarlane, H. Ohno, and B. Scrosati, "Ionic-liquid materials for the electrochemical challenges of the future," Nat. Mater., vol. 8, no. 8, pp. 621-629, 2009.

14 T. Welton, "Room-temperature ionic liquids. solvents for synthesis and catalysis," Chem. Rev., vol. 99, no. 8, pp. 2071-2084, 1999.

15 J. P. Hallett and T. Welton, "Room-temperature ionic liquids: solvents for synthesis and catalysis. 2," Chem. Rev., vol. 111, no. 5, pp. 3508-3576, 2011.

16 A. Goduljan, F. Juarez, L. Mohammadzadeh, P. Quaino, E. Santos, and W. Schmickler, "Screening of ions in carbon and gold nanotubes - a theoretical study," Electrochem. Comm., vol. 45, pp. 48-51, 2014. 
17 L. Mohammadzadeh, A. Goduljan, F. Juarez, P. Quaino, E. Santos, and W. Schmickler, "Nanotubes for charge storage - towards an atomistic model," Electrochim. Acta, vol. 162, pp. 11-16, 2015.

18 A. A. Kornyshev, "The simplest model of charge storage in single file metallic nanopores," Farad. Discuss., vol. 164, pp. 117-133, 2013.

19 A. A. Lee, S. Kondrat, and A. A. Kornyshev, "Single-file charge storage in conducting nanopores," Phys. Rev. Lett., vol. 113, no. 4, p. 048701, 2014.

20 V. Démery, D. S. Dean, T. C. Hammant, R. R. Horgan, and R. Podgornik, "The one-dimensional Coulomb lattice fluid capacitor," J. Chem. Phys., vol. 137, no. 6, p. 064901, 2012.

21 V. Démery, D. S. Dean, T. C. Hammant, R. R. Horgan, and R. Podgornik, "Overscreening in a 1D lattice Coulomb gas model of ionic liquids," Europhys. Lett., vol. 97, no. 2, p. 28004, 2012.

22 W. K. H. Panofsky and M. Phillips, Classical electricity and magnetism. Courier Corporation, 2005.

23 M. Blume, V. J. Emery, and R. B. Griffiths, "Ising model for the $\lambda$ transition and phase separation in $\mathrm{He}^{3}-\mathrm{He}^{4}$ mixtures," Phys. Rev. A, vol. 4, no. 3, p. 1071, 1971.

24 O. K. Smith, "Eigenvalues of a symmetric $3 \times 3$ matrix," Commun. ACM, vol. 4, no. 4, pp. 168-, 1961.

25 J. Kopp, "Efficient numerical diagonalization of hermitian $3 \times 3$ matrices," Int. J. Mod. Phys. $C$, vol. 19, no. 03, pp. 523-548, 2008.

26 C. J. Bouwkamp and N. G. De Bruijn, "The electrostatic field of a point charge inside a cylinder, in connection with wave guide theory," J. Appl. Phys., vol. 18, no. 6, pp. 562-577, 1947.

27 J. M. Griffin, A. C. Forse, H. Wang, N. M. Trease, P.-L. Taberna, P. Simon, and C. P. Grey, "Ion counting in supercapacitor electrodes using NMR spectroscopy," Faraday Discuss., vol. 176, pp. 49-68, 2014.

28 J. M. Griffin, A. C. Forse, W.-Y. Tsai, P.-L. Taberna, P. Simon, and C. P. Grey, "In situ NMR and electrochemical quartz crystal microbalance techniques reveal the structure of the electrical double layer in supercapacitors," Nat. Mater., vol. 14, p. 812, 2015.

29 A. C. Forse, J. M. Griffin, C. Merlet, P. M. Bayley, H. Wang, P. Simon, and C. P. Grey, "NMR study of ion dynamics and charge storage in ionic liquid supercapacitors," J. Am. Chem. Soc., vol. 137, pp. 7231-7242, 2015.

30 "MCCCS Towhee." http://towhee.sourceforge.net. Accessed: 22 Sep 2015. 
31 M. G. Martin, "MCCCS Towhee: a tool for Monte Carlo molecular simulation," Mol. Simul., vol. 39, no. 14-15, pp. 1212-1222, 2013.

32 B. Widom, "Some topics in the theory of fluids," J. Chem. Phys., vol. 39, no. 11, pp. 2808-2812, 1963.

33 S. Kondrat and A. Kornyshev, "Pressing a spring: What does it take to maximize the energy storage in nanoporous supercapacitors?," Nanoscale Horiz., vol. 1, pp. 45-52, 2016.

34 J. F. Jover, R. Lugo, H. Toulhoat, P. Simon, and T. De Bruin, "Screening methodology for the efficient pairing of ionic liquids and carbonaceous electrodes applied to electric energy storage," J. Phys. Chem. C, vol. 118, no. 2, pp. 864-872, 2014.

35 For the set of parameters used in the figures, the values of $h$ beyond the selected range result in fully saturated behaviour, which is of little interest to show. This is a specificity of ultra-narrow pores studied in our work, where the Coulomb interaction are almost completely screened and allow easy packing of counterions of the same size and decoupling of counterions and coions. For more ionophobic pores (currently not experimentally available) the region of saturation will be shifted to higher voltages. This may require a wider window for solvent molecules not to take part in any electrochemical reactions.

36 S. Kondrat, P. Wu, R. Qiao, and A. Kornyshev, "Accelerating charging dynamics in subnanometre pores," Nat. Mater., vol. 13, pp. 387-393 (7pp), 2014.

37 D. Jiang and J. Wu, "Unusual effects of solvent polarity on capacitance for organic electrolytes in a nanoporous electrode," Nanoscale, vol. 6, p. 5545, 2014.

38 D. Jiang, Z. Jin, and J. Wu, "Oscillation of capacitance inside nanopores," Nano Lett., vol. 11, no. 12 , pp. 5373-5377, 2011.

39 K. Kiyohara, T. Sugino, and K. Asaka, "Phase transition in porous electrodes," J. Chem. Phys., vol. 134, no. 15, p. 154710, 2011.

40 W. Schmickler, "A simple model for charge storage in a nanotube," Electochim. Acta, vol. 173, pp. 91-95, 2015.

41 A. A. Lee, S. Kondrat, G. Oshanin, and A. A. Kornyshev, "Charging dynamics of supercapacitors with narrow cylindrical nanopores," Nanotechnology, vol. 25, p. 315401, 2014.

42 S. Kondrat and A. Kornyshev, "Charging dynamics and optimization of nano-porous supercapacitors," J. Phys. Chem. C, vol. 117, pp. 12399-12406, 2013. 
43 H. Wang, A. C. Forse, J. M. Griffin, N. M. Trease, L. Trognko, P.-L. Taberna, P. Simon, and C. P. Grey, "In situ NMR spectroscopy of supercapacitors: Insight into the charge storage mechanism," J. Am. Chem. Soc., vol. 135, p. 18968, 2013.

44 M. D. Levi, G. S. N. Levy, D. Aurbach, and J. Maier, "Application of a quartz-crystal microbalance to measure ionic fluxes in microporous carbons for energy storage," Nat. Mater., vol. 8, p. 872,2009 .

45 M. D. Levi, N. Levy, S. Sigalov, G. Salitra, D. Aurbach, and J. Maier, "Electrochemical quartz crystal microbalance (eqcm) studies of ions and solvents insertion into highly porous activated carbons," J. Am. Chem. Soc., vol. 132, no. 38, pp. 13220-13222, 2010. PMID: 20828121.

46 S. Sigalov, M. D. Levi, L. Daikhin, G. Salitra, and D. Aurbach, "Electrochemical quartz crystal admittance studies of ion adsorption on nanoporous composite carbon electrodes in aprotic solutions," J. Solid State Electr., vol. 18, no. 5, pp. 1335-1344, 2013.

47 W.-Y. Tsai, R. Lin, S. Murali, L. L. Zhang, J. K. McDonough, R. S. Ruoff, P.-L. Taberna, Y. Gogotsi, and P. Simon, "Outstanding performance of activated graphene based supercapacitors in ionic liquid electrolyte from -50 to $80{ }^{\circ}$ c," Nano Energy, vol. 2, pp. 403-411, 2013.

48 W.-Y. Tsai, P.-L. Taberna, and P. Simon, "Electrochemical quartz crystal microbalance (EQCM) study of ion dynamics in nanoporous carbons.," J. Am. Chem. Soc., vol. 136, p. 8722, 2014.

49 D. Mattia and Y. Gogotsi, "Review: Static and dynamic behavior of liquids inside carbon nanotubes," Microfluid Nanofluid, vol. 5, no. 3, pp. 289-305, 2008. 
1

2

3

4

5

6

7

8

9

10

11

12

13

14

15

16

17

18

19

20

21

22

23

24

25

26

27

28

29

30

31

32

33

34

35

36

37

38

39

40

41

42

43

44

45

46

47

48

49

50

51

52

53

54

55

56

57

58

59

60

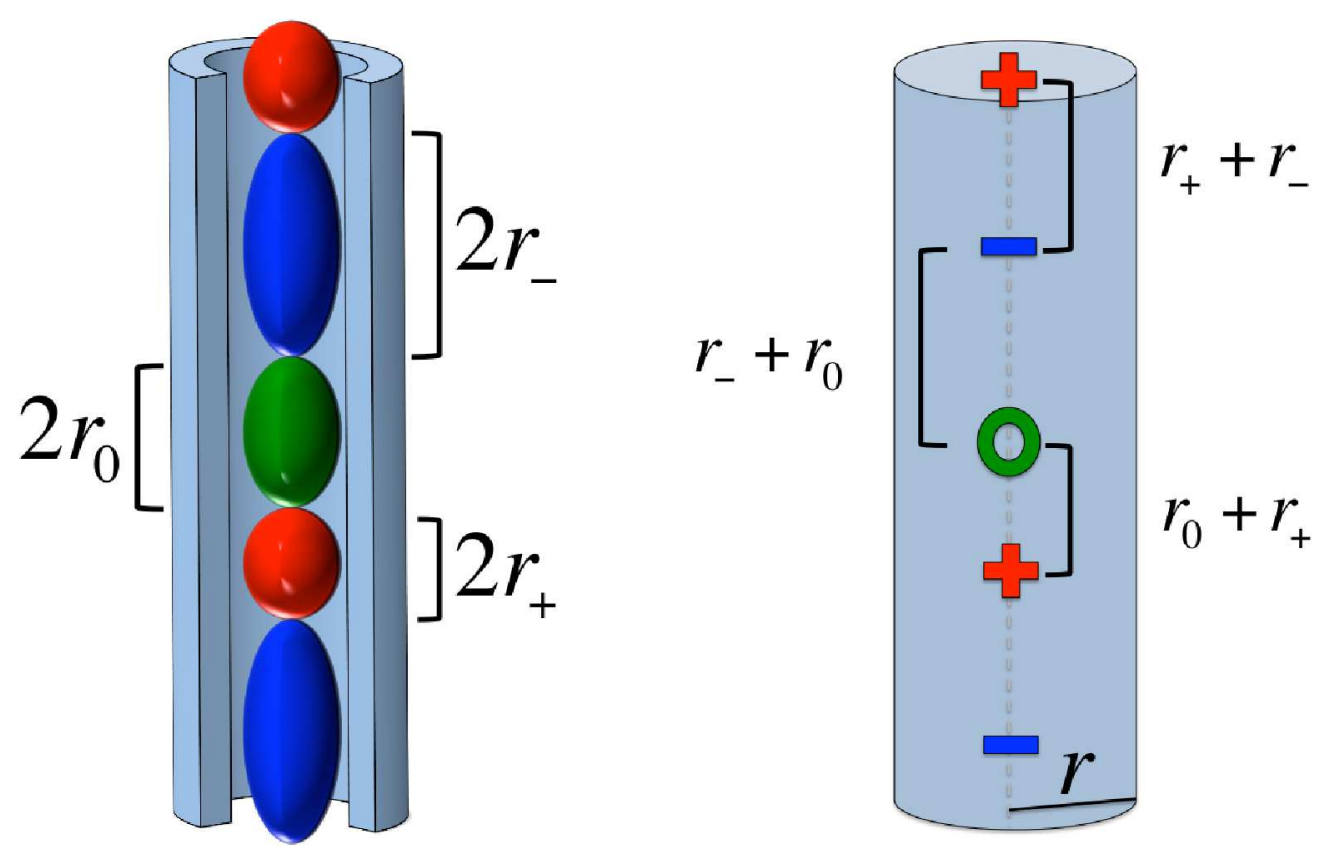

